\title{
Properties of convective delivery in spinal cord gray matter: laboratory investigation and computational simulations
}

\author{
Toshiki Endo, MD, PhD, ${ }^{1}$ Yushi Fujii, ${ }^{1}$ Shin-ichiro Sugiyama, MD, PhD, ${ }^{1}$ Rong Zhang, MD, PhD, ${ }^{1}$ \\ Shogo Ogita, MD, ${ }^{1}$ Kenichi Funamoto, PhD, ${ }^{2}$ Ryuta Saito, MD, PhD, ${ }^{1}$ and Teiji Tominaga, MD, PhD ${ }^{1}$ \\ 1Department of Neurosurgery, Graduate School of Medicine, and ${ }^{2}$ Creative Flow Research Division, Institute of Fluid Science, \\ Tohoku University, Sendai, Japan
}

\begin{abstract}
OBJECTIVE Convection-enhanced delivery (CED) is a method for distributing small and large molecules locally into the interstitial space of the spinal cord. Delivering these molecules to the spinal cord is otherwise difficult due to the bloodspinal cord barrier. Previous research has proven the efficacy of CED for delivering molecules over long distances along the white matter tracts in the spinal cord. Conversely, the characteristics of CED for delivering molecules to the gray matter of the spinal cord remain unknown. The purpose of this study was to reveal regional distribution of macromolecules in the gray and white matter of the spinal cord with special attention to the differences between the gray and white matter.

METHODS Sixteen rats (F344) underwent Evans blue dye CED to either the white matter (dorsal column, 8 rats) or the gray matter (ventral horn, 8 rats) of the spinal cord. The rates and total volumes of infusion were $0.2 \mu \mathrm{l} / \mathrm{min}$ and $2.0 \mu \mathrm{l}$, respectively. The infused volume of distribution was visualized and quantified histologically. Computational models of the rat spinal cord were also obtained to perform CED simulations in the white and gray matter.

RESULTS The ratio of the volume of distribution to the volume of infusion in the gray matter of the spinal cord was 3.60 \pm 0.69 , which was comparable to that of the white matter $(3.05 \pm 0.88)$. When molecules were injected into the white matter, drugs remained in the white matter tract and rarely infused into the adjacent gray matter. Conversely, when drugs were injected into the gray matter, they infiltrated laterally into the white matter tract and traveled longitudinally and preferably along the white matter. In the infusion center, the areas were larger in the gray matter CED than in the white matter (Mann-Whitney U-test, $p<0.01$ ). In computational simulations, the aforementioned characteristics of CED to the gray and white matter were reaffirmed.
\end{abstract}

CONCLUSIONS In the spinal cord, the gray and white matter have distinct characteristics of drug distribution by CED. These differences between the gray and white matter should be taken into account when considering drug delivery to the spinal cord. Computational simulation is a useful tool for predicting drug distributions in the normal spinal cord.

http://thejns.org/doi/abs/10.3171/2015.5.SPINE141148

KEY WORDS computational simulation; convection-enhanced delivery; gray matter; spinal cord; technique; white matter

$\mathrm{N}$ UMEROUS therapeutic compounds are now available for treating CNS disorders. However, only select agents can penetrate the blood-brain or blood-spinal cord barriers and reach the targeted regions to exert clinical effects. We have used a technique for delivering an anticancer drug regionally to the brain and distributing the drug in the brain using convection in the interstitial space, i.e., convection-enhanced delivery (CED) ${ }^{20,25}$
Convective delivery of therapeutic substances to the spinal cord has also been postulated elsewhere. ${ }^{12,14,27} \mathrm{CED}$ to the spinal cord provides a reproducible, safe, regionspecific, and homogeneous distribution of macromolecules over large longitudinal segments of the spinal cord. ${ }^{12}$ To date, the characteristics of drug distribution by CED have been demonstrated exclusively in the white matter of the spinal cord. Conversely, little is known regarding drug

ABBREVIATIONS ALS = amyotrophic lateral sclerosis; $\mathrm{CED}=$ convection-enhanced delivery; $\mathrm{Vd}=$ volume of distribution; $\mathrm{Vi}=$ volume of infusion .

SUBMITTED November 13, 2014. ACCEPTED May 20, 2015.

INCLUDE WHEN CITING Published online October 30, 2015; DOI: 10.3171/2015.5.SPINE141148. 
distribution in the gray matter. It is important to establish the CED technique in both the gray and white matter of the spinal cord, particularly when we consider treating diseases related to changes in the gray matter, such as amyotrophic lateral sclerosis (ALS). In ALS, drugs should reach the anterior horn of the gray matter, where degenerated motor neurons are located. ${ }^{8}$

In this report, we performed CED of Evans blue dye to the gray and white matter of the normal rodent spinal cord. We intended to reveal the characteristics of CED to the gray matter of the spinal cord with the aim of performing selective drug delivery to the gray matter. Moreover, we constructed computational models of the spinal cord and performed computational simulation of CED to the gray and white matter. The results of simulations were compared with those analyzed histologically.

\section{Methods}

\section{Animal Preparation}

Twenty-three male adult F344 rats (Charles River Laboratories Japan, Inc.) weighing approximately $200 \mathrm{~g}$ were used in this study. They received a volume of infusion (Vi) of $2.0 \mu \mathrm{l}$ of Evans blue dye (E2129-10G, Sigma-Aldrich Japan) either into the dorsal columns $(n=8)$ or ventral horn of the gray matter $(n=8)$ of the T-9 thoracic spinal cord. Six animals were used to confirm location of the injection. One animal was used for model reconstruction in computer simulation. All animal investigations were conducted in accordance with the guidelines and ethical protocols approved by the Institute for Animal Experimentation of Tohoku University Graduate School of Medicine.

\section{Preparation of Evans Blue Dye and Delivery System}

Evans blue dye was diluted to a concentration of $4.0 \%$ in sterile phosphate-buffered saline (Wako Pure Chemical Industries) for all infusions. To deliver Evans blue dye to the spinal cord by CED, we modified a system that we had reported previously. ${ }^{19,24,25}$ The infusion system consisted of a reflux-free step-design infusion cannula. ${ }^{9,12}$ A flexible silica tube (inner diameter, $100 \mu \mathrm{m}$; outer diameter, $170 \mu \mathrm{m}$; Polymicro Technologies, LLC) was fused with a 27-gauge cannula needle (Terumo Medical Corp.; Fig. 1). The needle was glued and connected to a loading line (containing Evans blue) and an infusion line (filled with oil). A 1-ml syringe, filled with oil, was mounted onto a microinfusion pump (BeeHive, Bioanalytical Systems) to regulate the flow of fluid through the system. The infusion cannula was mounted onto stereotactic holders and guided to the target region of the spinal cord through laminectomy. The cannula was inserted into the dorsal spinal cord at an angle of $45^{\circ}$ in the cranial direction. We intended to advance the cannula in an angled manner rather than perpendicular to the spinal cord axis, aiming to reduce the backflow of Evans blue dye based on our preliminary experiences (data not included).

\section{Evans Blue Dye CED to the Rat Spinal Cord}

The rats were anesthetized with isoflurane anesthesia $(2.0 \%)$ while lying on a heating pad. The heating pad was adjusted to maintain the temperature of the rats at $35.5^{\circ} \mathrm{C}-$

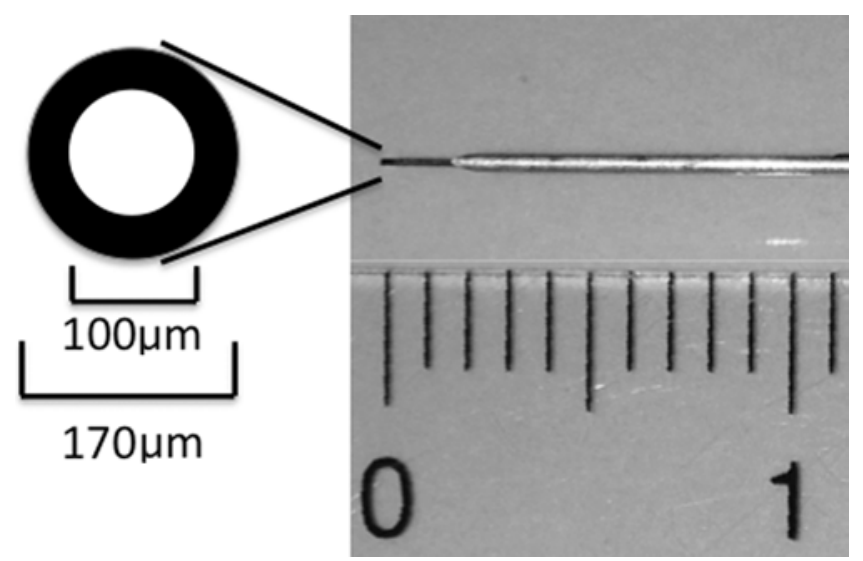

FIG. 1. Illustration and photograph exhibiting the infusion cannula used for spinal cord CED. Left: Inner and outer diameters of a silica tube were $100 \mu \mathrm{m}$ and $170 \mu \mathrm{m}$, respectively. Right: The tip of a flexible silica tube protruded $2 \mathrm{~mm}$ from a 27-gauge cannula needle, which was suitable for CED to the gray matter.

$37.5^{\circ} \mathrm{C}$ during surgery. Hair was clipped from each animal's back, and the site of incision was disinfected. Surgery was performed using a sterile technique with the aid of a Zeiss OPMI-6 surgical microscope (Carl Zeiss Meditec Co., Ltd.). A 2-cm midline incision was made along the T-8 to T-10 spinal processes. Following laminectomy of T-9, the dura mater was exposed and left intact. Following exposure, rats were secured using a small animal stereotactic frame (David Kopf Instrument). Spinal processes were held in place to ensure that the spinal cord did not move due to respiration (Fig. 2A). Care was taken to place the rats' spinal cord parallel to the horizontal plane. With the aid of an operating microscope, the dura was punctured with the tip of a 27-gauge needle. Through the hole, the silica infusion cannula was advanced at an angle of $45^{\circ}$ as described previously (Fig. 2B). The silica cannula was trimmed at lengths of $1.4 \mathrm{~mm}$ and $2.0 \mathrm{~mm}$ for CED to the white and gray matter, respectively. For CED to the white matter, the cannula penetrated the pia mater in the midline immediately next to the dorsal vein. The cannula was advanced $1.4 \mathrm{~mm}$ to reach a depth of $1.0 \mathrm{~mm}$ at the center of the dorsal column of the spinal cord. ${ }^{1}$ For CED to the gray matter, the cannula pierced the pia mater $0.8 \mathrm{~mm}$ laterally to the midline and advanced $2.0 \mathrm{~mm}$ to reach the gray matter, which was located at a depth of 1.4 $\mathrm{mm}$ from the dorsal surface of the spinal cord according to the stereotactic atlas (Fig. 2C). ${ }^{16}$ The infusion rate of Evans blue dye was $0.2 \mu \mathrm{l} / \mathrm{min}$, which led to a total Vi of $2.0 \mu \mathrm{l}$ after 10 minutes in either the gray or white matter of experimental animals. Five minutes after the completion of the infusion, the cannula was slowly removed.

\section{Confirmation of Injection Locations}

We injected Shandon Tissue-Marking Dye (Thermo Fisher Scientific) into 6 separate animals to confirm whether a tip of the cannula reached a designated area in the spinal cord. After we inserted a needle either in the gray $(n=3)$ or the white matter $(n=3)$ in the aforementioned way, we delivered Tissue-Marking Dyes for 30 seconds $(0.2 \mu \mathrm{l} / \mathrm{min})$. Immediately after the injections, animals 

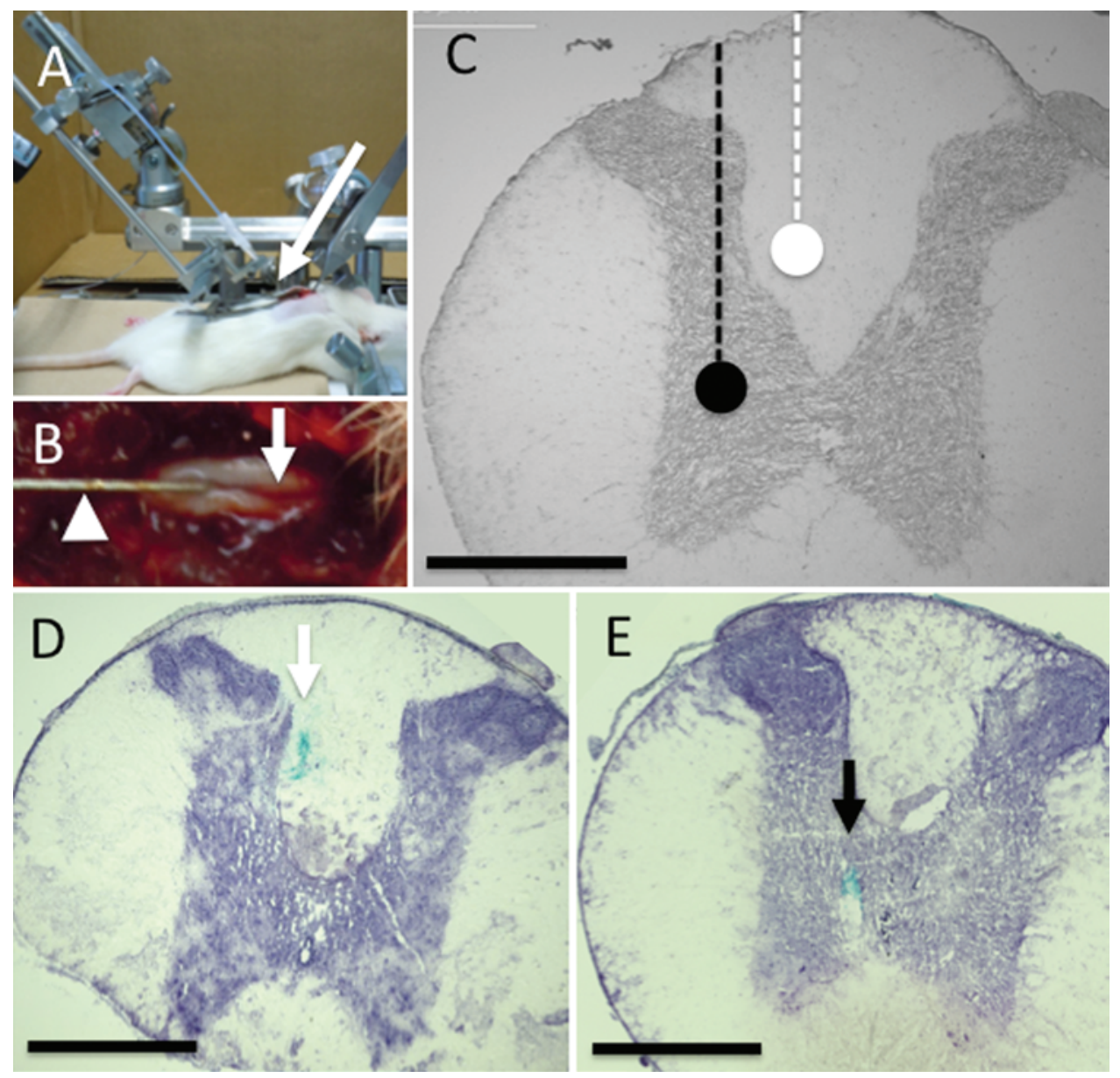

FIG. 2. A photograph and a schema illustrating spinal cord CED. A: A rat is placed in a stereotactic head frame and held by forceps. Note that the needle (arrow) is inserted at an angle of $45^{\circ}$ degrees to the spinal cord. B: A silica infusion cannula is inserted into the dorsal spinal cord without injury to the dorsal vein (arrow). A 27-gauge needle is observed (arrowhead). However, a silica cannula is already embedded in the spinal cord parenchyma. C: A schema showing the planned positions of infusion cannula in a representative axial spinal cord slice. The black and white dots are located in the gray and white matter, respectively. The depths from the dorsal surface of the spinal cord are $1.4 \mathrm{~mm}$ (black) and $1.0 \mathrm{~mm}$ (white). D and E: Axial histological sections of the spinal cord with hematoxylin stain showing Tissue-Marking Dye at injection locations in the white (D, white arrow) and the gray matter (E, black arrow). Bars $=800 \mu \mathrm{m}$. Figure is available in color online only.

were humanely killed with an overdose of barbiturate. The spinal cord segment including the site of injections was removed for histological analyses. It was frozen and sliced into 20- $\mu \mathrm{m}$ axial sections for hematoxylin staining. In this experiment, injection locations were confirmed by detecting Tissue-Marking Dye by a light microscope.

\section{Histological Analysis and Measurement of Evans Blue Dye Distribution}

Immediately after CED, animals were humanely killed with an overdose of barbiturate for histological analysis. Using the T-9 segment as a center, a length of $5 \mathrm{~cm}$ of the spinal cords was removed. The spinal cord was cut transversely at the site of infusion and divided into rostral and caudal segments. Rostral and caudal spinal cord sections were further divided into 3 segments each. All 6 segments were frozen and sliced into $20-\mu \mathrm{m}$ axial sections for $\mathrm{H}$ \& E staining. By preparing histological specimens in this way, we were able to mark the infusion point. The areas infiltrated by Evans blue dye were confirmed using a light microscope, and every 5 sections were measured manually using image analysis software (ImageJ 1.47, NIH). The volume of distributions ( $\mathrm{Vd}$ ) was calculated by multiplying the areas by $0.1 \mathrm{~mm}$. For statistical analysis, data between the gray and white matter were compared using the Mann-Whitney U-test.

\section{Model Reconstruction and Meshing for Computational Simulation}

For computer simulation, the thoracic spinal cord was excised from 1 rat and scanned to acquire an MR image. A scanner with a 5-cm bore operating at 9.4-T field strengths (AV400WB, Bruker BioSpin) was used with the following parameters: FOV $20 \mathrm{~mm} \times 5 \mathrm{~mm} \times 5 \mathrm{~mm}, 512 \times$ $128 \times 128$ matrix, TR/TE $24 \mathrm{msec} / 10 \mathrm{msec} / 30,6$ averages. Scan time was 24 minutes. The surface geometry of the rodent spinal cord was reconstructed semiautomatically based on the tissue intensity using stacks of T1-weighted MR images. Anatomically consistent geometry of the white and gray matter was separated manually, and tri- 
angulated surface meshes were generated using imaging software (Avizo 6.2, Visualization Sciences Group). The spinal cord model including the white and gray matter was meshed using commercial software (ICEM CFD; ANSYS Inc.). The infusion site (radius, $0.1 \mathrm{~mm}$ ) was incorporated into the white or gray matter. The final tetrahedral mesh contained approximately 640,000 elements. The domain includes fine meshes for the catheter tip to acquire precise computational simulation.

\section{Simulation of Drug Infusion}

Drug infusion into the spinal cord by CED was modeled as previously reported. ${ }^{23}$ Please see supplementary information for details of methodology regarding computer simulations (Supplementary Materials and Methods) The baseline values of the parameters used in this study are summarized in Table 1.22

Following the computer simulation, drug distribution was determined separately in the white and gray matter. The threshold of volume fractions of the Evans blue dye was set at $10 \%$, according to our previous report. ${ }^{23}$ The results of computer simulations were compared with those of histological analysis using the Pearson correlation coefficient test.

\section{Results}

\section{Injection Locations in the Gray and the White Matter CED}

Using Tissue-Marking Dye, we histologically confirmed a location of the cannula tip in the gray and the white matter ( $\mathrm{n}=3$ each). In 6 consecutive animals, our protocol was consistently successful in placing the needle in designated areas for spinal cord CED. Representative histological sections are provided (Fig. 2D and E).

\section{Distribution of Evans Blue Dye in the Spinal Cord}

After infusion of Evans blue dye into the rat spinal cord, the ratios of the volume of distribution to the volume of infusion $(\mathrm{Vd} / \mathrm{Vi})$ were $3.60 \pm 0.69$ and $3.05 \pm 0.88$ in the gray and white matter, respectively. There was no

TABLE 1. Baseline parameters used in computational CED simulations in this study

\begin{tabular}{ll}
\hline \multicolumn{1}{c}{ Parameter } & Value \\
\hline Porosity & 0.26 \\
\hline Hydraulic conductivity $\left(\mathrm{cm}^{4} /\right.$ dyne $\left.\bullet \mathrm{sec}\right)$ & \\
\hline Gray matter & $4.22 \times 10^{-12}$ \\
\hline $\mathrm{K}_{\text {parallel }}, \mathrm{K}_{\text {vertical }}$ & \\
\hline White matter & $6.75 \times 10^{-9}$ \\
\hline $\mathrm{K}_{\text {parallel }}$ & $4.22 \times 10^{-10}$ \\
\hline $\mathrm{K}_{\text {vertical }}$ & \\
\hline Density $\left(\mathrm{kg} / \mathrm{m}^{3}\right)$ & 1002 \\
\hline $\mathrm{CSF}$ & 1042 \\
\hline Evans blue $(4 \%)$ & \\
\hline Viscosity $(\mathrm{mPa} \cdot \mathrm{sec})$ & 1.003 \\
\hline Water & 0.1 \\
\hline Catheter diameter $(\mathrm{mm})$ &
\end{tabular}

statistical difference between $\mathrm{Vd} / \mathrm{Vi}$ of the gray and white matter. Importantly, in CED to the white matter, Evans blue dye remained in the dorsal column, and it was distributed longitudinally (Fig. 3). In CED to the gray matter, the dye did not remain in the gray matter. Rather, the agent preferentially infiltrated the white matter and traveled longitudinally along the white matter tracts (Fig. 4). Figure 5 presents the areas of Evans blue dye distribution. In CED to the gray matter, Evans blue dye was distributed in an area of $3.04 \pm 0.44 \mathrm{~mm}^{2}$ around the site of injection, which was significantly larger than that in CED to white matter $\left(2.02 \pm 0.60 \mathrm{~mm}^{2}, \mathrm{p}<0.01\right)$. Conversely, concerning the longitudinal length of distribution, CED to the white matter showed a distribution length of $7.87 \pm 2.16 \mathrm{~mm}$, which was longer than that shown by CED to the gray matter $(5.26 \pm 1.41 \mathrm{~mm})$, and this difference was statistically significant $(\mathrm{p}=0.02)$.

\section{Computational Simulation of CED to the Spinal Cord}

In the computational models of the spinal cord, CED to the white matter delivered the hypothetical Evans blue dye preferentially in the craniocaudal direction along the white matter tracts (Fig. 6 upper). The distribution of the blue dye was limited within the white matter. In contrast, in CED to the gray matter, the dye infiltrated into the white matter tracts. Consequently, the dye was distributed horizontally over larger areas at the level of infusion, and the agent traveled longitudinally along the white matter tracts (Fig. 6 lower). In CED to the gray matter, Evans blue dye traveled shorter distances longitudinally than those observed in CED to the white matter. Computational simulations reproduced the results obtained in the experimental studies, as shown in Figs. 3-5. Pearson correlation coefficient values of white and gray matter between the computer simulation and the histological analysis were $0.75(\mathrm{p}$ $<0.001)$ and $0.88(\mathrm{p}<0.001)$, respectively.

\section{Discussion}

CED is a local infusion technique that uses positive pressure to deliver therapeutic agents to the CNS. ${ }^{3,4}$ This method can infuse small or large molecules into targeted sites homogeneously in larger volumes than possible with simple diffusion. ${ }^{3,10,11}$ Having used nimustine hydrochloride CED, we demonstrated its efficacy in treating highgrade intracranial glioma in experimental animals and patients..$^{20,21,24,25}$ In CED, therapeutic agents can pass through the blood-brain barrier and reach the target areas. Thus, the technique is less likely to cause systemic side effects that could be a problem in systemic administration. ${ }^{6,26}$

\section{CED to the White Matter of the Spinal Cord}

To date, CED has been mainly applied to brain pathology, and reports regarding CED to the spinal cord are limited. However, Lonser et al. described that the white matter tracts of the spinal cord provide ideal conditions for CED..$^{12,14}$ Namely, convective flow in the spinal cord is anisotropic and runs parallel to the fiber tracts.,18 Thus, longitudinal drug delivery along the white matter tracts was achievable in the rodent and primate spinal cord. ${ }^{12}$ In the current study, we applied CED to white matter in the 


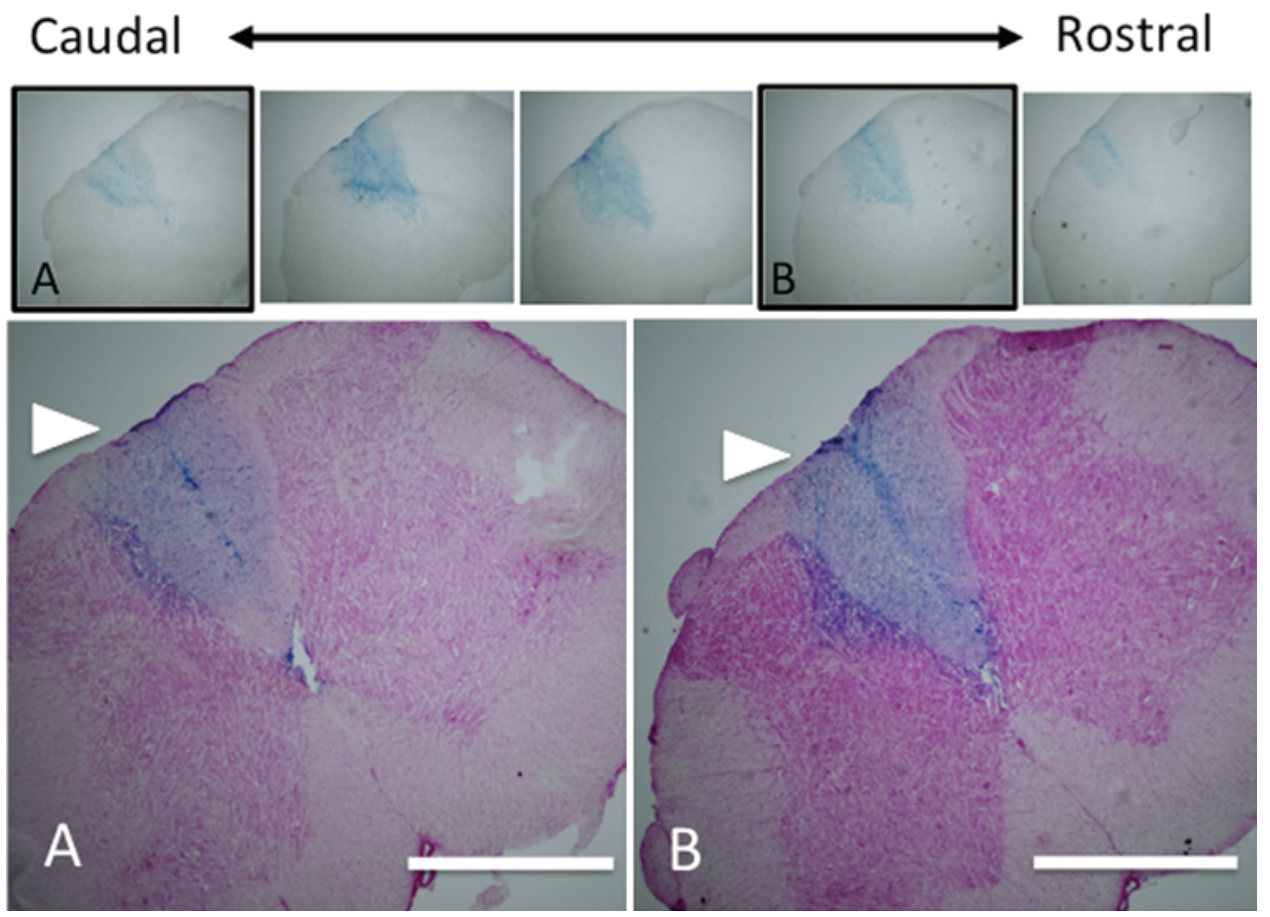

FIG. 3. Evans blue dye CED to the white matter. Upper: Axial histological sections of the spinal cord were placed from the rostral (right) to the caudal (left) direction. Lower: Magnified images of A and B with $\mathrm{H}$ \& $\mathrm{E}$ stain, demonstrating the characteristics of CED to the white matter. Note that Evans blue dye remained in the dorsal column spinal cord (arrowheads) and spread longitudinally. Bars $=800 \mu \mathrm{m}$. Figure is available in color online only.

spinal cords of healthy rodents. The $\mathrm{Vd} / \mathrm{Vi}$ ratio was 3.05 \pm 0.88 in the white matter, which was comparable to that reported previously. ${ }^{12,14}$ Importantly, the infused materials did not infiltrate into the adjacent gray matter. Rather, they stayed in the white matter tracts, i.e., dorsal columns, and traveled preferentially in longitudinal directions (Fig. 3).
This makes CED to the white matter a useful tool for delivering drugs over multiple spinal levels along the tracts. At the same time, based on our results, we inferred that CED to the white matter has limited efficacy, because drugs rarely reached the ventral spinal cord when injected into the dorsal column.

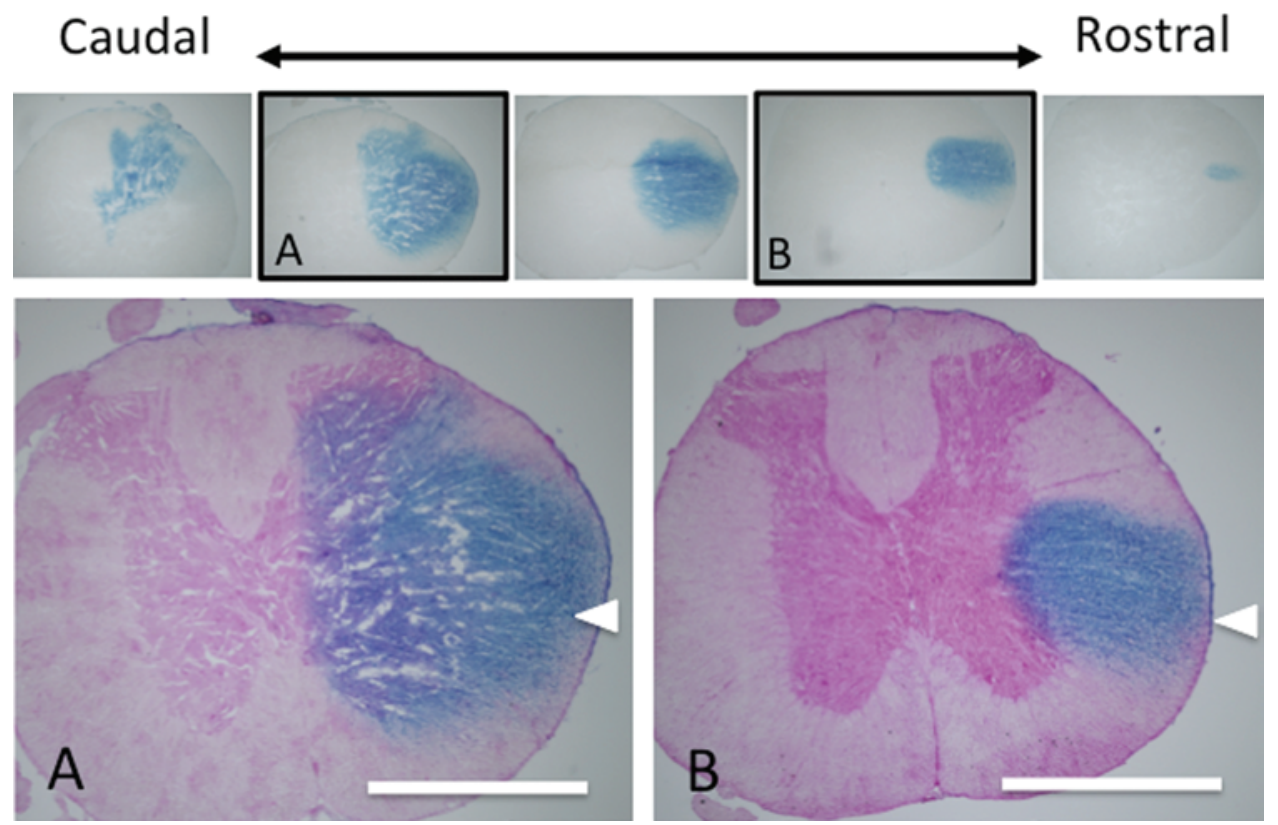

FIG. 4. CED to the gray matter. Upper: Axial sections were placed as described in Fig. 3. Lower: Magnified images of A and B with $\mathrm{H} \& \mathrm{E}$ stain, demonstrating the characteristics of CED to the gray matter, in which Evans blue dye infiltrated into the lateral white matter tracts (arrowheads) from the gray matter. Bars $=800 \mu \mathrm{m}$. Figure is available in color online only. 


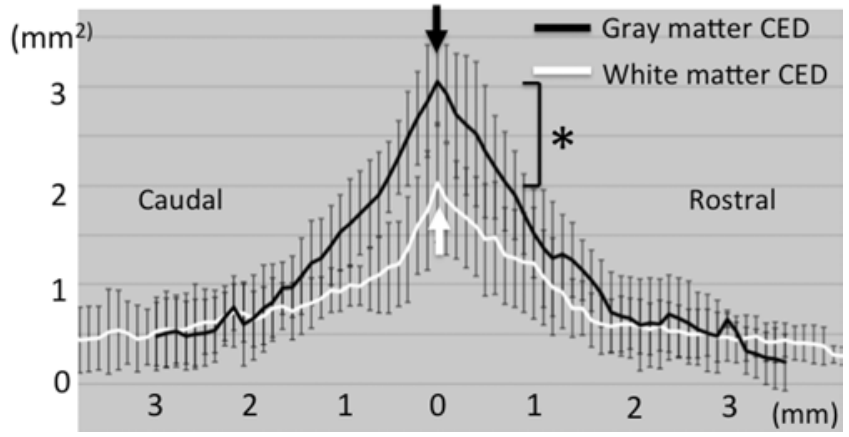

FIG. 5. Diagram showing the distribution of Evans blue dye in CED to the gray (black line) and white (white line) matter. Arrows indicate injection points. Numbers on the $\mathrm{X}$-axis indicate distances $(\mathrm{mm})$ from the injection points. Numbers on the $y$-axis indicate areas of distributed Evans blue dye. Note that the dye was distributed over longer distances in the rostral and caudal directions in CED to the white matter than in CED to the gray matter. Conversely, the dye was distributed to wider areas around the infusion centers in CED to the gray matter. ${ }^{*} p<0.01$.

\section{CED to the Gray Matter of the Spinal Cord}

In this study, we performed CED to the gray matter. The $\mathrm{Vd} / \mathrm{Vi}$ ratio of CED to the gray matter was $3.60 \pm 0.69$, which was not statistically different from that of CED to the white matter. However, the drug distribution pattern of CED to the gray matter was distinct. Notably, the infused Evans blue dye was not restricted to the gray matter. Rather, the agent infiltrated into the white matter and traveled along the white matter tracts in the lateral spinal cord. Consequently, in the infusion center of CED to the gray matter, the agent was distributed in larger areas of the spinal cord, covering both the gray and white matter in the axial plane. Regarding traveling longitudinally in the craniocaudal direction, infused materials traveled shorter distances in the gray matter than those observed with CED to the white matter. This indicated that CED to the gray matter is suitable when considering drug delivery to cover large horizontal spinal cord areas in limited vertebral levels.

\section{Computational CED Simulation}

Computational simulation of CED to the spinal cord reproduced the aforementioned experimental observations. Namely, in the white matter, the dye was distributed exclusively in the white matter and traveled longer distances longitudinally. In contrast, in the gray matter, the dye infiltrated into the adjacent white matter and traveled along the white matter (Fig. 6). As reported previously, drug distribution in the spinal cord may strongly rely on structural differences. ${ }^{17}$ Sarntinoranont et al. described the microscopic structure of the spinal cord using diffusionweighted MRI, ${ }^{22}$ which revealed greater fractional anisotropy in the white matter along the tracts. Hydraulic conductivity in the gray matter was lower, which resulted in increased tissue resistance in the gray matter. This would explain why drugs easily penetrated into the white matter where tissue resistance is lower, when it was injected from the gray matter. Our computational models of CED to the gray matter faithfully demonstrated this hypothetical drug infiltration. Computational simulation is noteworthy, because it clearly demonstrated the differences between CED to the gray and white matter in the rat spinal cord.
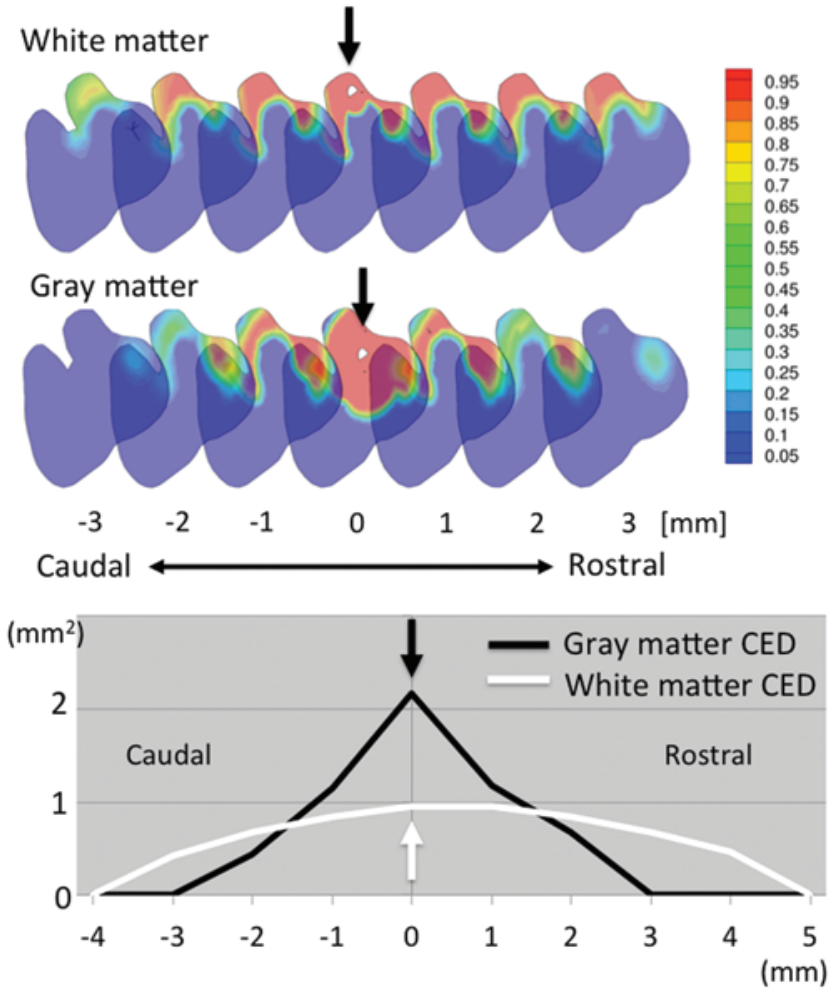

FIG. 6. Computational simulation of Evans blue dye in CED to the rat spinal cord (2.0 $\mu$ f for $10 \mathrm{~min})$. Upper: In CED to the white matter, the dye was distributed exclusively in the white matter. In CED to the gray matter, the dye was distributed horizontally in the white matter and traveled longitudinally. Numbers on the $x$-axis indicate distances from the injection points. The color bar indicates the volume fraction of Evans blue dye (from 0 to 1). The injection points are indicated by arrows. Lower: Diagram illustrating the distribution of Evans blue dye in CED to the gray (black line) and white (white line) matter as predicted by computational simulation. Arrows and numbers on the $\mathrm{x}$ - and $\mathrm{y}$-axes indicate distances and areas as described in Fig. 5. Note the characteristics of CED computer simulation are similar to those of the actual spinal cord experiments. Figure is available in color online only.

\section{Clinical Applications}

When we consider drug infiltration in patients with spinal cord disorders, the differences between CED to the white and gray matter are important. Prediction of the distribution areas of therapeutic materials plays an important role in the performance of spinal cord CED in a safe and reliable manner. ${ }^{23}$ For instance, when we intended to deliver drugs into the gray matter of the spinal cord, CED into the dorsal column may not have been ideal. From the current results, it is expected that drugs are less likely to infiltrate into the gray matter from the white matter. More specifically, if we want to deliver a neurotrophic factor into the motor neurons in the anterior horn of patients with ALS, CED to the gray matter should be a treatment of choice. Computational simulation can possibly identify the ideal location of infusion as well as the rate and timing of infusions for the best possible outcomes.

In the case of clinical application of CED, safety is also important. Because CED distributes therapeutic agents through extracellular spaces by hydrostatic pressure, it is less likely to cause local tissue damage., ${ }^{3,13}$ Nonetheless, 
when we treat intramedullary tumors by CED, care should be taken because an anticancer drug itself may induce local tissue toxicity. ${ }^{25}$ By using animal models, our group was able to determine maximum tolerable concentrations of nimustine hydrochloride ${ }^{25}$ or doxorubicin, ${ }^{28}$ which were not hazardous to neural and neuronal tissues in rat striatum. Based on these preclinical studies, we have organized protocols of nimustine hydrochloride CED to treat clinically challenging brainstem glioma. ${ }^{20,21}$

The optimal treatment strategy for malignant spinal cord glioma remains to be established. ${ }^{7}$ Attempting complete surgical excisions is difficult, because the surgical dissection plane is not clear in most intramedullary spinal cord astocytomas. ${ }^{5,15}$ In such cases, it would be intriguing if CED infused anticancer drugs through interstitial spaces in the spinal cord without causing new neurological deficits to patients. For that purpose, it is mandatory that concentrations of anticancer drugs should not exceed potentially toxic ranges. Before clinical application, each cytotoxic agent should be examined in experimental studies to disclose therapeutic as well as toxic concentrations in the spinal cord.

The limitations of this study include that our findings are solely limited to the healthy spinal cord. Efforts to apply this method to spinal cord injury models are extremely important, as postulated elsewhere. ${ }^{27}$ If we could control the distribution areas and amount of drugs delivered, this technique should be useful to improve clinical outcomes in patients with spinal cord diseases.

\section{Conclusions}

In CED to the spinal cord, the gray and white matter have distinct characteristics of drug distribution. These differences between the gray and white matter are important for delivering therapeutic materials to the spinal cord in a safe and reliable manner. Computational simulation is a useful and feasible option for predicting drug distribution when CED to the spinal cord is performed.

\section{Acknowledgments}

This work was supported in part by a research grant from The General Insurance Association of Japan, Grant-in-Aid for Scientific Research (C) (KAKENHI 24592151), and Grant-in-Aid for Young Scientists (B) (KAKENHI 24791483). We thank Misaki Fue and Haruka Mitome for their excellent technical assistance.

\section{References}

1. Anderson KD, Gunawan A, Steward O: Spinal pathways involved in the control of forelimb motor function in rats. Exp Neurol 206:318-331, 2007

2. Basser PJ, Pierpaoli C: Microstructural and physiological features of tissues elucidated by quantitative-diffusion-tensor MRI. J Magn Reson B 111:209-219, 1996

3. Bobo RH, Laske DW, Akbasak A, Morrison PF, Dedrick RL, Oldfield EH: Convection-enhanced delivery of macromolecules in the brain. Proc Natl Acad Sci U S A 91:2076-2080, 1994

4. Chen MY, Lonser RR, Morrison PF, Governale LS, Oldfield EH: Variables affecting convection-enhanced delivery to the striatum: a systematic examination of rate of infusion, cannula size, infusate concentration, and tissue-cannula sealing time. J Neurosurg 90:315-320, 1999
5. Garcés-Ambrossi GL, McGirt MJ, Mehta VA, Sciubba DM, Witham TF, Bydon A, et al: Factors associated with progression-free survival and long-term neurological outcome after resection of intramedullary spinal cord tumors: analysis of 101 consecutive cases. J Neurosurg Spine 11:591-599, 2009

6. Groothuis DR: The blood-brain and blood-tumor barriers: a review of strategies for increasing drug delivery. Neuro Oncol 2:45-59, 2000

7. Hsu S, Quattrone M, Ostrom Q, Ryken TC, Sloan AE, Barnholtz-Sloan JS: Incidence patterns for primary malignant spinal cord gliomas: a Surveillance, Epidemiology, and End Results study. J Neurosurg Spine 14:742-747, 2011

8. Ishigaki A, Aoki M, Nagai M, Warita H, Kato S, Kato M, et al: Intrathecal delivery of hepatocyte growth factor from amyotrophic lateral sclerosis onset suppresses disease progression in rat amyotrophic lateral sclerosis model. J Neuropathol Exp Neurol 66:1037-1044, 2007

9. Krauze MT, Saito R, Noble C, Tamas M, Bringas J, Park JW, et al: Reflux-free cannula for convection-enhanced highspeed delivery of therapeutic agents. J Neurosurg 103:923929, 2005

10. Laske DW, Morrison PF, Lieberman DM, Corthesy ME, Reynolds JC, Stewart-Henney PA, et al: Chronic interstitial infusion of protein to primate brain: determination of drug distribution and clearance with single-photon emission computerized tomography imaging. J Neurosurg 87:586-594, 1997

11. Lieberman DM, Laske DW, Morrison PF, Bankiewicz KS, Oldfield EH: Convection-enhanced distribution of large molecules in gray matter during interstitial drug infusion. J Neurosurg 82:1021-1029, 1995

12. Lonser RR, Gogate N, Morrison PF, Wood JD, Oldfield EH: Direct convective delivery of macromolecules to the spinal cord. J Neurosurg 89:616-622, 1998

13. Lonser RR, Sarntinoranont M, Morrison PF, Oldfield EH: Convection-enhanced delivery to the central nervous system. J Neurosurg 122:697-706, 2015

14. Lonser RR, Walbridge S, Garmestani K, Butman JA, Walters HA, Vortmeyer AO, et al: Successful and safe perfusion of the primate brainstem: in vivo magnetic resonance imaging of macromolecular distribution during infusion. J Neurosurg 97:905-913, 2002

15. McGirt MJ, Goldstein IM, Chaichana KL, Tobias ME, Kothbauer KF, Jallo GI: Extent of surgical resection of malignant astrocytomas of the spinal cord: outcome analysis of 35 patients. Neurosurgery 63:55-61, 2008

16. Paxinos G, Watson C: The Rat Brain in Stereotaxic Coordinates, The New Coronal Set, ed 5. New York: Academic Press, 2005

17. Prokopová S, Vargová L, Syková E: Heterogeneous and anisotropic diffusion in the developing rat spinal cord. Neuroreport 8:3527-3532, 1997

18. Reulen HJ, Graham R, Spatz M, Klatzo I: Role of pressure gradients and bulk flow in dynamics of vasogenic brain edema. J Neurosurg 46:24-35, 1977

19. Saito R, Krauze MT, Bringas JR, Noble C, McKnight TR, Jackson P, et al: Gadolinium-loaded liposomes allow for realtime magnetic resonance imaging of convection-enhanced delivery in the primate brain. Exp Neurol 196:381-389, 2005

20. Saito R, Sonoda Y, Kumabe T, Nagamatsu K, Watanabe M, Tominaga T: Regression of recurrent glioblastoma infiltrating the brainstem after convection-enhanced delivery of nimustine hydrochloride. J Neurosurg Pediatr 7:522-526, 2011

21. Saito R, Tominaga T: Convection-enhanced delivery: from mechanisms to clinical drug delivery for diseases of the central nervous system. Neurol Med Chir (Tokyo) 52:531-538, 2012 
22. Sarntinoranont M, Chen X, Zhao J, Mareci TH: Computational model of interstitial transport in the spinal cord using diffusion tensor imaging. Ann Biomed Eng 34:1304-1321, 2006

23. Sugiyama S, Saito R, Funamoto K, Nakayama T, Sonoda Y, Yamashita Y, et al: Computational simulation of convectionenhanced drug delivery in the non-human primate brainstem: a simple model predicting the drug distribution. Neurol Res 35:773-781, 2013

24. Sugiyama S, Saito R, Nakamura T, Yamashita Y, Yokosawa M, Sonoda Y, et al: Safety and feasibility of convectionenhanced delivery of nimustine hydrochloride co-infused with free gadolinium for real-time monitoring in the primate brain. Neurol Res 34:581-587, 2012

25. Sugiyama S, Yamashita Y, Kikuchi T, Saito R, Kumabe T, Tominaga T: Safety and efficacy of convection-enhanced delivery of ACNU, a hydrophilic nitrosourea, in intracranial brain tumor models. J Neurooncol 82:41-47, 2007

26. Walter KA, Tamargo RJ, Olivi A, Burger PC, Brem H: Intratumoral chemotherapy. Neurosurgery 37:1128-1145, 1995

27. Wood JD, Lonser RR, Gogate N, Morrison PF, Oldfield EH: Convective delivery of macromolecules into the naive and traumatized spinal cords of rats. J Neurosurg 90 (1 Suppl):115-120, 1999

28. Zhang R, Saito R, Mano Y, Kanamori M, Sonoda Y, Kumabe $\mathrm{T}$, et al: Concentration rather than dose defines the local brain toxicity of agents that are effectively distributed by convection-enhanced delivery. J Neurosci Methods 222:131-137, 2014

\section{Disclosure}

The authors report no conflict of interest concerning the materials or methods used in this study or the findings specified in this paper.

\section{Author Contributions}

Conception and design: Endo, Sugiyama, Saito, Tominaga. Acquisition of data: Endo, Fujii, Sugiyama, Zhang, Ogita. Analysis and interpretation of data: Endo, Sugiyama, Zhang, Funamoto. Drafting the article: Endo, Fujii, Sugiyama, Ogita. Critically revising the article: Funamoto, Tominaga. Reviewed submitted version of manuscript: all authors. Approved the final version of the manuscript on behalf of all authors: Endo. Statistical analysis: Endo, Sugiyama, Zhang, Funamoto. Administrative/technical/material support: Saito, Tominaga. Study supervision: Saito, Tominaga.

\section{Supplemental Information}

\section{Online-Only Content}

Supplemental material is available with the online version of the article.

Supplementary Materials and Methods. http://thejns.org/doi/ suppl/10.3171/2015.5.SPINE141148.

\section{Correspondence}

Toshiki Endo, Department of Neurosurgery, Graduate School of Medicine, Tohoku University, 1-1 Seiryo Aoba, Sendai 9808574, Japan. email: endo@nsg.med.tohoku.ac.jp. 